Nloman 2015, 33(2), 67-75

Revista de Psicologia, Ciències de l'Educació i de l'Esport

ISSN: 1138-3194

Copyright (C) 2015

www.revistaaloma.net

\title{
Adicción a los videojuegos en línea: tratamiento mediante el programa PIPATIC
}

\author{
Alexandra Torres-Rodríguez \& Xavier Carbonell \\ Universitat Ramon Llull \\ Recibido: 16-6-2015 \\ Aceptado: 9-9-2015
}

Adicción a los videojuegos en línea: tratamiento mediante el programa PIPATIC

Resumen. El desarrollo de las Tecnologías de la Información y la Comunicación (TIC) ha propiciado investigaciones sobre la adicción a Internet, a los videojuegos y teléfonos móviles y su tratamiento. El objetivo del presente estudio es presentar la aplicación de un programa de tratamiento psicoterapéutico para la adicción a las TIC (PIPATIC) y la evaluación de su efectividad en un estudio de caso único de un paciente con trastorno dual: Internet Gaming Disorder (IGD) y trastorno obsesivo-compulsivo (TOC). Los resultados son alentadores y muestran cambios positivos: reducción significativa del tiempo dedicado a los juegos, aumento del autocontrol y reducción de la sintomatología general y de los síntomas relacionados con el TOC.

Palabras clave: trastorno de juego por Internet; trastorno obsesivo-compulsivo; adicción a videojuegos; patología dual; tratamiento; estudio de caso

Online videogame addiction: Treatment using the PIPATIC program

Summary. The development of Information and Communication Technology (CIT) has given rise to research on addictions to the Internet, videogames and mobile phones and their treatment. The objective of this study is to apply a program of psychotherapeutical treatment for CIT addiction (PIPATIC) and to evaluate its effectiveness via a single case study of a patient with a dual pathology: Internet Gaming Disorder (IGD) and Obsessive Compulsive Disorder (OCD). The results are encouraging and display positive changes: a significant reduction in the time spent on games, an increase in self-control and a reduction in overall symptomology and in the symptoms related to $O C D$.

Keywords: Internet Gaming Disorder; Obsessive Compulsive Disorder; videogame disorder; dual pathology; treatment; case study

Correspondencia

Alexandra Torres-Rodríguez

Facultat de Psicologia, Ciències de l'Educació i de l'Esport

Blanquerna

Universitat Ramon Llull

c/Cister, 34

08022 Barcelona

Email: alexandrart@blanquerna.edu 


\section{Introducción}

Las Tecnologías de la Información y la Comunicación (TIC) destacan por su importancia socioeconómica y popularidad, en especial internet, los móviles y los videojuegos, ya que han provocado importantes cambios conductuales. En la población española, diversos informes (Fundación Orange, 2014; ONTSI, 2013) coinciden en el aumento de personas que utilizan estas tecnologías. En cuanto a internet, el número de personas, entre 16 a 74 años, que se conectan diariamente ha alcanzado la cifra de 18.651.864 (ONTSI, 2013). Por otro lado, un 96\% de los hogares españoles tiene teléfonos móviles (Fundación Orange, 2014). Asimismo, cabe destacar la importancia de los videojuegos, siendo el segundo contenido digital de pago más importante de España (Fundación Orange, 2014). Según la Asociación de Distribuidores y Editores de Software de Entretenimiento (ADESE, 2009), en España hay 10,4 millones de videojugadores de los que 7,2 millones son jugadores de consola y 6,7 millones juegan en su ordenador personal. Es por ello que la comunidad científica se ha interesado por las repercusiones provocadas por el uso desadaptativo de las TIC, especialmente, por las adicciones tecnológicas (Carbonell, Fúster, Chamarro \& Oberst, 2012; Carbonell, Guardiola, Beranuy \& Bellés, 2009; Griffiths \& Meredith, 2009; King \& Delfabbro, 2014) y se han publicado algunos estudios de casos (Alario, 2006; Allison, Wahlde, Shockley, \& Gabbard, 2006; Beranuy et al., 2012; Griffiths, 2000; Marco \& Chóliz, 2014). De estas tecnologías, los videojuegos en línea son los que provocan más uso desadaptativo (Carbonell et al., 2012; González, Merino \& Cano, 2009; Kelly, 2004).

Cabe remarcar que la mayoría de los videojuegos permiten jugar en línea con otros usuarios, esto hace que los videojuegos de consola y ordenador de diversos géneros se parezcan cada vez más a los Multiplayer Online Role-Playing Game (MMORPG) en cuanto a los mecanismos adictivos del juego: comunidades en línea en las que es posible relacionarse, elegir el modo de juego, videojuegos que no tienen final y «jugar cómo y cuándo quieras durante el tiempo que desees». Yee (2006) detectó que un 40\% de usuarios de MMORPG se consideraban a sí mismos como «adictos». Los jugadores tienen diversas motivaciones como socializarse, explorar, conseguir logros y disociarse de los problemas o de la realidad y se ha hipotetizado que el uso problemático podría producirse cuando la identidad se ve alterada y la importancia de la identidad virtual supera a la identidad real (Fuster et al., 2012).

El impacto de los videojuegos en línea ha culminado con la incorporación del Internet Gaming Disorder (IGD), traducido al español como trastorno de juego por internet, descrito en la sección III del DSM-5 como un trastorno que necesita más estudio (American Psychiatric Association, 2013). En relación a ello, se ha intentado demostrar que los criterios propuestos para el IGD tienen una adecuada validez y precisión diagnóstica (Ko et al., 2014).
Desde el punto de vista clínico, los estudios publicados sugieren que ha habido un incremento de la demanda de tratamiento de las adicciones tecnológicas, tanto en poblaciones infantojuveniles como adultas (Beranuy, Carbonell, \& Griffiths, 2012; González et al., 2009; Matalí \& Alda, 2008). En cuanto a su tratamiento, el enfoque más aceptado es el cognitivo-conductual (Greenfield, 1999; Griffiths \& Meredith, 2009; Young, 2007, 2011, 2013), proponiendo utilizar técnicas aplicadas en otras adicciones: entrevista motivacional, control de estímulos, balance decisional, autorregistros, técnicas de tolerancia al malestar, reestructuración cognitiva, etc. Asimismo, Young $(2011,2013)$ ha desarrollado una terapia cognitivo-conductual específica para la adicción a internet (CBT-IA). Por lo tanto, se propone el tratamiento cognitivo-conductual específico con los mismos principios que se han aplicado a otras adicciones (King, Delfabbro, Griffiths, \& Gradisar, 2011). La dificultad de tratamiento recae en que las adicciones tecnológicas están asociadas en numerosas ocasiones a otros trastornos mentales y de personalidad (Chappell, Eatough, Davies, \& Griffiths, 2006; Cruzado, Matos, \& Kendall, 2006; Ferguson, Coulson, \& Barnett, 2011; Gentile et al., 2011; Han, Lee, Shi, \& Renshaw, 2014; Shapira, Goldsmith, Keck Jr, Khosla, \& McElroy, 2000; Yen et al., 2008). El propósito de este estudio es exponer un caso clínico de IGD que presenta diversas comorbilidades.

\section{Método}

\section{Diseño del estudio y procedimiento}

El éste presente estudio se caracteriza por ser la aplicación piloto del programa de intervención psicológica para el uso problemático de tecnologías de la información y la comunicación (Torres, Carbonell, \& Oberst, manuscrito no publicado) diseñado con el objetivo de tratar las adicciones tecnológicas y, especialmente, el IGD. El proceso se inició con una primera entrevista informativa en la que se comunicó al paciente los objetivos, el modelo de intervención y las premisas éticas de confidencialidad y voluntariedad del programa y se cumplimentó el consentimiento informado. El objetivo del tratamiento es: a) reducir la sintomatología clínica respecto al inicio del tratamiento (en los síntomas clínicos, hábitos de uso desasaptativos y problemas de conducta); y b) mejorar las siguientes competencias: recursos psicológicos, autoestima, inteligencia emocional, habilidades sociales, clima familiar y funcionamiento global.

\section{Presentación del caso: historia y evolución del problema}

M es un varón de 40 años, separado con un hijo pequeño, que acude de forma voluntaria en busca de ayuda psicológica para superar su adicción a los videojuegos en línea. El motivo de la demanda de M es: «estoy aquí porque quiero dejar de jugar a los videojuegos, me he dado cuenta de que estoy muy enganchado. 
Los videojuegos me han llevado a una ruptura sentimental y a perder mi trabajo». Tenía buena predisposición al tratamiento y motivación. M refería que hace unos años ya había acudido a un tratamiento sin recibir la ayuda que esperaba, lo cual era indicador de mal pronóstico terapéutico. En la primera visita, M explicó que su malestar se agravó a partir de un duelo y una pérdida de empleo que generaron una crisis vital. Atribuyó la pérdida del empleo a su adicción a los videojuegos en línea, ya que no cumplía los objetivos de ventas al jugar en horario laboral. Una vez sin empleo, mentía reiteradamente a su pareja sobre el tiempo invertido en los videojuegos y sobre las entrevistas de trabajo a las que supuestamente acudía. Finalmente, la sintomatología adictiva provocó la ruptura con su pareja y se fue a vivir con sus padres. A pesar de las numerosas consecuencias negativas que provocaba el uso desapatativo de los videojuegos y de los intentos fallidos de reducir las horas de juego por sí mismo, se mostraba incapaz de plantearse dejar de jugar: «no puedo, no voy a poder nunca», encontrándose, por tanto, en una etapa de cambio precontemplativa (Prochaska, Norcross, \& DiClemente, 1994). El elevado malestar y los recientes eventos vitales le empujaban a la necesidad de cambiar, a pesar de ello no era capaz de hacer nada al respecto. El contexto familiar de $\mathrm{M}$ en su infancia se caracterizó por un estilo educativo permisivo (Baumrind, 1968), sin límites ni consecuencias sobre conductas negativas (por ejemplo, salir excesivamente por la noche, no estudiar, jugar a videojuegos todas las horas que quisiera, poner un candado en la habitación, etc.). $\mathrm{M}$ refiere tener una sintomatología adictiva, ansiosa, depresiva y obsesivo-compulsiva, además de pensamientos bizarros, conductas agresivas e irritables, anhedonia, falta de energía y descuido en la higiene personal. Al verbalizar todo ello se produjo en él la siguiente reflexión «a veces lo pienso y creo que estoy fatal de la cabeza, estoy loco». Además, en las primeras entrevistas diagnósticas se observaba una baja autoestima, inteligencia emocional y capacidad de adaptación.

\section{Evaluación e instrumentos}

Puesto que M presentaba diversos cuadros sintomáticos se exploró la sintomatología adictiva, obsesivo-compulsiva, ansiosa y depresiva mediante entrevistas diagnósticas semiestructuradas y se administró la batería protocolizada del programa de intervención PIPATIC que contiene instrumentos autoaplicados y heteroaplicados (ver tabla 1).

\section{Diagnóstico}

Según los resultados e información obtenidos en el proceso de evaluación (tabla 2), los síntomas de $\mathrm{M}$ se adecuan a los criterios del DSM-5 (American Psychiatric Association, 2013) para el Internet Gaming Disorder: a) craving; b) aparición de sintomatología al dejar de jugar: irritabilidad al interrumpir el juego; c) tolerancia: «cada vez necesito jugar más para ser mejor»; d) intentos de control fallidos: «el otro día intenté dejarlo pero no pude, me fue imposible, creo que necesito que me ayuden a dejarlo porque solo no puedo»; e) pérdida de intereses: «no hay nada que me dé placer, no hay ninguna otra actividad que me guste, aunque antes me encantaba correr»; f) uso excesivo y continuado, a pesar de que los videojuegos le generen una problemática importante: pérdida de empleo y ruptura de pareja; g) ha provocado una decepción en las personas allegadas, lo que ha causado poner en riesgo relaciones significativas a causa del elevado tiempo invertido en los videojuegos; h) se usan los videojuegos para escapar o evitar estados emocionales negativos. Por otro lado, aunque M empezó a jugar a videojuegos cuando era un niño, mantenía una conducta de uso adecuada a pesar de que en el hogar había ausencia de límites, tenía otras áreas de interés, no estaba aislado socialmente y cumplía con sus responsabilidades; el problema se inició con la pérdida de un familiar. Cuando inició tratamiento $\mathrm{M}$ jugaba alrededor de 7 a 10 horas diarias, especialmente, a MMORPG y a Multiplayer Online Battle Arena (MOBA) y refería un importante malestar sintomático.

Tabla 1. Esquema de instrumentos de evaluación del programa PIPATIC: pretratamiento, monitorización, postratamiento y a los seis meses de finalizar el tratamiento

\begin{tabular}{|c|c|}
\hline Evaluación pretratamiento & $\begin{array}{l}\text { - Cuestionario sobre los hábitos de uso de videojuegos (elaboración propia) } \\
\text { - Cuestionario de Experiencias Relacionadas con Internet (CERI), Cuestionario de Experiencias Relacionadas } \\
\text { con el Móvil (CERM), Cuestionario de Experiencias Relacionadas con los Videojuegos (CERV) de Beranuy, } \\
\text { Chamarro, Graner, y Carbonell (2009) } \\
\text { - Inventario Clínico Multiaxial de Millon (MCMI-III) de Millon, Millon, Davis, y Grossman (2009) } \\
\text { - SCL-90-R: cuestionario de } 90 \text { síntomas de Derogatis (2002) } \\
\text { - Escala Rasgo de Metaconocimientos sobre Estados Emocionales (TMMS-24): Trait Meta-Mood Scale (Salovey, } \\
\text { Mayer, Goldman, Turvey, \& Palfai, 1995) versión española de (Fernández-Berrocal, Alcaide, Domínguez, } \\
\text { Fernández-McNally, Ramos \& Ravira, 1998) } \\
\text { - EHS Escala de Habilidades Sociales (Gismero, 2000) } \\
\text { - Escala de Clima Social Familiar (FES) (Moos, 1974) } \\
\text { - Escala de Impresión Clínica Global para severidad de la enfermedad (ICG) } \\
\text { - Escala de Evaluación de la Actividad Global-EEAG (American Psychiatric Association, 1987) }\end{array}$ \\
\hline Monitorización del tratamiento & $\begin{array}{l}\text { - Cuestionario sobre los hábitos de uso de videojuegos (elaboración propia) } \\
\text { - SCL-90-R } \\
\text { - EEAG } \\
\text { - ICG }\end{array}$ \\
\hline Evaluación Postratamiento & $\begin{array}{l}\text { Conformada por la misma batería inicial, añadiendo lo siguiente: } \\
\text { - Working Alliance Theory of Change Inventory (WATOCI): versión en español (Corbella \& Botella, 2004) }\end{array}$ \\
\hline Evaluación a los seis meses & $\begin{array}{l}\text { Conformada por: Cuestionario sobre los hábitos de uso de videojuegos, CERI, CERM, CERV, MCMI-III, SCL-90-R, } \\
\text { ICG, EEAG }\end{array}$ \\
\hline
\end{tabular}


Tabla 2. Puntuaciones de los instrumentos de las baterías de evaluación del tratamiento

\begin{tabular}{|c|c|c|c|c|}
\hline & & Pretratamiento & Monitorización & Postratamiento \\
\hline CERV & & 50 & & 3 \\
\hline \multirow{3}{*}{ TMMS-24 } & Atención & 20 & & 17 \\
\hline & Claridad & 19 & & 40 \\
\hline & Reparación & 14 & & 40 \\
\hline EHS Total & & 75 & & 99 \\
\hline CGS-I & & 5 & & 1 \\
\hline EEAG & & 41 & & 70 \\
\hline \multirow{12}{*}{ SCL-90-R } & Somatización & 0.41 & 0 & 0 \\
\hline & Obsesivo-compulsivo & 2.1 & 1.2 & 0.6 \\
\hline & Sensibilidad interpersonal & 1.66 & 0.33 & 0 \\
\hline & Depresión & 2.53 & 0.53 & 0.53 \\
\hline & Ansiedad & 1.3 & 0.4 & 0.4 \\
\hline & Hostilidad & 1.16 & 0.33 & 0.3 \\
\hline & Ansiedad fóbica & 1.14 & 0.28 & 0.1 \\
\hline & Ideación paranoide & 0.66 & 0 & 0 \\
\hline & Psicoticismo & 1.2 & 0 & 0.1 \\
\hline & Ítems adicionales & 4 & 2 & 0 \\
\hline & GSI & 1.35 & 0.35 & 0.24 \\
\hline & PST & 51 & 26 & 19 \\
\hline \multirow{16}{*}{ MCMI-III* } & Esquizoide (1) & 68 & & 32 \\
\hline & Evitativo (2A) & 76 & & 11 \\
\hline & Depresivo (2B) & 70 & & 2 \\
\hline & Dependiente (3) & 60 & & 15 \\
\hline & Antisocial (6A) & 83 & & 64 \\
\hline & Agresivo (6B) & 67 & & 9 \\
\hline & Negativista (8A) & 63 & & 15 \\
\hline & Autodestructivo (8B) & 62 & & 2 \\
\hline & Esquizotípico (S) & 64 & & 2 \\
\hline & Límite (C) & 62 & & 2 \\
\hline & Tr. de ansiedad (A) & 80 & & 32 \\
\hline & Tr. distímico (D) & 68 & & 2 \\
\hline & Dependencia del alcohol (B) & 78 & & 32 \\
\hline & Dependencia de sustancias (T) & 68 & & 62 \\
\hline & Tr. de estrés postraumático (R) & 62 & & 2 \\
\hline & Depresión mayor (CC) & 60 & & 2 \\
\hline
\end{tabular}

Nota: * Se reflejan las puntuaciones superiores a 60 puntos en el pretratamiento puesto que según el manual de MCMI-III (Millon et al., 2009) los 60 puntos indicarían la mediana pudiendo considerarse las puntuaciones obtenidas como rasgos.

En referencia a los trastornos comórbidos de este caso, según resultados obtenidos por los instrumentos aplicados (tabla 1) y las entrevistas semiestructuradas, los síntomas presentados por $\mathrm{M}$ son compatibles con un trastorno obsesivo-compulsivo (ver tabla 2). M recuerda que en su adolescencia tenía una manía con un cenicero que siempre debía estar en el mismo lugar; actualmente, presenta obsesiones (miedo a que algo malo ocurra a su familia, compulsiones y conductas de comprobación). M es consciente de que sus obsesiones son ilógicas y mantiene las compulsiones para reducir el malestar que le causan, estas también se desencadenaron después del duelo traumático. Por otro lado, en cuanto a la personalidad, M muestra rasgos de personalidad antisocial y evitativa (ver tabla 2). Otras características de la personalidad del participante son: baja autoestima, inseguridad social, baja tolerancia a la frustración, elevada impulsividad, baja empatía, incorrecta gestión emocional, falta de recursos de afrontamiento, etc. Asimismo, otro problema que puede ser objeto de atención clínica es una ruptura familiar por separación y un duelo complicado. Es conveniente destacar que realizaba un consumo de alcohol excesivo los fines de semana hasta que conoció a su pareja (trastorno por consumo de alcohol en remisión). En cuanto a los recursos psicológicos que presenta, destacamos las buenas habilidades sociales a pesar de que, internamente, se muestra inseguro socialmente. Inicialmente, $\mathrm{M}$ reflejaba dificultades para generar insight por si mismo, pero una vez que el terapeuta lo promovía $\mathrm{M}$ era capaz iniciar el proceso de reflexión y mentalización, lo que promovía generar consciencia del malestar psicológico y colaboración en la terapia.

\section{El programa PIPATIC}

El programa PIPATIC se compone de seis módulos, a su vez están formados por áreas de trabajo psicoterapéutico más específicas (ver tabla 3), distribuidos en una sesión informativa y 22 sesiones semanales de 45 
Tabla 3. Programa PIPATIC: composición por módulos de trabajo psicoterapéutico

1. Módulo psicoeducativo: psicoeducación, psicoeducación familiar, entrevista motivacional, definición de metas y objetivos. Aplicación en tres sesiones.

2. Módulo de intervención estándar en adicciones conductuales: control de estímulos en la adicción, aprendizaje de respuestas de afrontamiento adecuadas, reestructuración cognitiva, solución de problemas relacionados con la adicción y exposición. Aplicación en seis sesiones.

3. Módulo intrapersonal: fomentar la identidad, la autoestima, el control de los impulsos, las emociones y la ansiedad, las habilidades de afrontamiento y la solución de problemas intrapersonales. Aplicación en cinco sesiones.

4. Módulo interpersonal: fomentar capacidades adaptativas de comunicación, generar asertividad, modificar el estilo de respuesta social. Aplicación en dos sesiones.

5. Módulo familiar: comunicación familiar, límites familiares y vínculos afectivos. Aplicación en tres sesiones.

6. Módulo de creación de un nuevo estilo de vida: autoobservación de Módulo de creación de un nuevo estilo de vida: autoobservación de
la mejoría, actividades alternativas y prevención de recaídas. Aplicación en dos sesiones.

minutos; en total, unos seis meses de tratamiento, una duración según los estándares recomendados (Kadera, Lambert, \& Andrews, 1996; Lambert \& Bergin, 1994). El objetivo del PIPATIC es tratar las adicciones tecnológicas y los trastornos o problemáticas comórbidas. La intervención está centrada en: a) la orientación psicoterapéutica cognitivo-conductual; b) la integración de diversas orientaciones psicológicas utilizando técnicas y recursos terapéuticos propios de estas; y c) aplicar a lo largo del tratamiento una serie de recursos terapéuticos y técnicas psicológicas comunes en psicoterapia (Kleinke, 1995) de forma transversal (independientemente del módulo de intervención psicoterapéutico): mostrar empatía, escucha activa y reflexiva, aceptación, confianza, grado intermedio de directividad, paráfrasis, clarificación, síntesis, confrontación, interpretación, proporcionar feedback, promover capacidades, promover la responsabilidad, generar sentimiento de autoeficacia, suscitar la capacidad de insight y fomentar la alianza terapéutica. Inicialmente, se utiliza la entrevista motivacional para generar una mayor alianza terapéutica y un compromiso con el tratamiento.

Los principales objetivos del tratamiento pactados con $\mathrm{M}$ fueron: el control de la conducta adictiva, adquirir autonomía y habilidades psicológicas adecuadas para afrontar dificultades y restablecer una salud psicológica normalizada y adaptativa. M no aceptó la participación de su madre ni la de su pareja. Expresaba que no quería preocuparlas porque quería avanzar por sí mismo en el proceso y solo permitió la participación de su pareja en una ocasión para el control de estímulos y la abstinencia.

En la tabla 4 se recogen ejemplos de diversos procesos de reestructuración cognitiva de los pensamientos irracionales nucleares de $\mathrm{M}$ y se utilizan las premisas de la terapia racional emotiva conductual de Ellis (1990a, 1990b; Lega, Caballo \& Ellis, 2009) y la terapia cognitiva de Beck (1979).

\section{Resultados del tratamiento}

Para medir la efectividad del tratamiento se registraron las ausencias, el cumplimiento del programa PIPATIC y el aprovechamiento de las sesiones. M acudió al 90\% de las visitas, aprovechó un 80\% las sesiones y cumplió el 90\% del programa terapéutico. En cuanto a la conducta de juego, hay que destacar que las elevadas puntuaciones en el CERV, en la autopuntuación subjetiva de adicción, en las horas de juego, en la frecuencia de conexión y en el aumento del autocontrol (ver tablas 2 y 5) se redujeron significativamente después de finalizar el tratamiento (ver apartado de postratamiento en las tablas 2 y 5 ).

Tabla 4. Procesos de reestructuración cognitiva en el tratamiento psicológico

\begin{tabular}{|c|c|c|c|c|}
\hline Acontecimiento (A) & $\begin{array}{l}\text { Creencias o pensamientos } \\
\text { irracionales (B) }\end{array}$ & $\begin{array}{l}\text { Conductas y/o emociones } \\
\text { (C) }\end{array}$ & $\begin{array}{l}\mathrm{N} .^{\circ} \text { de sesiones en las que } \\
\text { aparece y se trabaja la RC }\end{array}$ & $\begin{array}{l}\text { (D) Reestructuración } \\
\text { cognitiva (RC) }\end{array}$ \\
\hline Jugar al videojuego & $\begin{array}{l}\text { «Cuanto más juegue mejor } \\
\text { seré» }\end{array}$ & $\begin{array}{l}\text { Sentirme bien, aliviado, } \\
\text { con la autoestima alta } \\
\text { cuando gano }\end{array}$ & 5 & $\begin{array}{l}\text { «Cuanto más juegue para } \\
\text { ser mejor, me quedará } \\
\text { menos tiempo para hacer } \\
\text { cosas que son importantes } \\
\text { para mí» }\end{array}$ \\
\hline Ganar en el videojuego & $\begin{array}{l}\text { «Cuanto más gane más } \\
\text { subiré en el ranking y } \\
\text { querrá decir que soy 'pro'» }\end{array}$ & Autoestima que se eleva & 5 & $\begin{array}{l}\text { «Mi autoestima no debe } \\
\text { depender de si soy bueno } \\
\text { en el juego, mi autoestima } \\
\text { debe estar fuerte } \\
\text { independientemente» }\end{array}$ \\
\hline $\begin{array}{l}\text { Me olvidé de entregar un } \\
\text { paquete en el trabajo }\end{array}$ & «Me echarán del trabajo» & $\begin{array}{l}\text { Inseguridad, miedo al } \\
\text { fracaso, angustia }\end{array}$ & 1 & $\begin{array}{l}\text { «Todos somos humanos y } \\
\text { nos podemos equivocar, } \\
\text { pediré disculpas y estaré } \\
\text { más atento la próxima } \\
\text { vez» }\end{array}$ \\
\hline $\begin{array}{l}\text { Ir al parque con mi hijo y } \\
\text { relacionarme con los padres }\end{array}$ & $\begin{array}{l}\text { «Cuando quiero conocer a } \\
\text { un padre pienso que no le } \\
\text { caeré bien» }\end{array}$ & $\begin{array}{l}\text { Inseguridad, baja } \\
\text { autoestima }\end{array}$ & 6 & $\begin{array}{l}\text { «i eres amable con } \\
\text { alguien no tienes porqué } \\
\text { caerle mal, no puedo } \\
\text { anticipar algo que aún no } \\
\text { ha sucedido» }\end{array}$ \\
\hline $\begin{array}{l}\text { Durante muchos años he } \\
\text { estado en paro }\end{array}$ & $\begin{array}{l}\text { «Nunca encontraré un } \\
\text { trabajo estable» }\end{array}$ & Miedo, mucha inseguridad & 6 & $\begin{array}{l}\text { «puedo tener la } \\
\text { oportunidad de encontrar } \\
\text { un trabajo, nunca lo } \\
\text { encontraré si no hago el } \\
\text { esfuerzo de buscar» }\end{array}$ \\
\hline $\begin{array}{l}\text { Salgo de casa, toco algún } \\
\text { objeto }\end{array}$ & $\begin{array}{l}\text { «Algo malo va a suceder si } \\
\text { no hago el ritual» }\end{array}$ & $\begin{array}{l}\text { Realización de la conducta } \\
\text { obsesiva/ritual. } \\
\text { Frustración, sufrimiento y } \\
\text { angustia }\end{array}$ & 9 & $\begin{array}{l}\text { «Que sucedan cosas malas } \\
\text { no tiene ninguna relación } \\
\text { con mis manías, suceden } \\
\text { independientemente de si } \\
\text { las hago o no» }\end{array}$ \\
\hline
\end{tabular}


Tabla 5. Comparación de resultados del cuestionario sobre hábitos de uso de videojuegos

\begin{tabular}{lccc}
\hline & & \multicolumn{2}{c}{ Evaluación } \\
\cline { 2 - 4 } & Pretratamiento & Monitorización & Postratamiento \\
\hline $\begin{array}{l}\text { N. de horas diarias dedicadas al juego } \\
\text { para ver la evolución del juego }\end{array}$ & 10 horas & Ninguna & 0 \\
$\begin{array}{l}\text { Dificultad de aplazar o dejar de jugar al } \\
\text { videojuego }\end{array}$ & Elevada & Ninguna & Ninguna \\
$\begin{array}{l}\text { Autopercepción de adicción a los } \\
\text { videojuegos }\end{array}$ & Elevada & 3 & 2 \\
\hline
\end{tabular}

En la tabla 2, se pueden observar diversos aspectos como por ejemplo que en la evaluación pretratamiento en el instrumento TMMS-24 la puntuación indicaba baja inteligencia emocional: M prestaba poca atención a los sentimientos, comprendía mal sus estados emocionales y presentaba mala regulación emocional. M presentaba puntuaciones dentro de la normalidad en cuanto a las habilidades sociales (EHS). En el área de funcionamiento familiar (FES) las puntuaciones revelaban una problemática en los límites, en el control en el hogar y un excesivo interés en lo recreativo dejando de lado algunas responsabilidades. Las puntuaciones obtenidas en el SCL-90-R indicaban la presencia de: a) un elevado sufrimiento psicológico global; b) una actitud maximizadora frente a la sintomatología; y c) elevados síntomas de somatización, obsesivo-compulsivos, de sensibilidad interpersonal, de depresión, ansiedad, hostilidad y ansiedad fóbica. El perfil que sugiere el MCMI-III según las puntuaciones, era un patrón clínico de la personalidad antisocial, evitativo y depresivo y un patrón de síndromes clínicos de trastorno de ansiedad y de dependencia del alcohol.

Las puntuaciones postratamiento de la tabla 2 indican que la sintomatología que en el pretratamiento se mostraba alterada alcanza la normalidad en todos los campos, por ejemplo, en cuanto a la sintomatología en general medida con el SCL-90-R al final del tratamiento se encontraron que las puntuaciones descendían a baremos dentro de la normalidad. Como M no respondió el FES en la batería postratamiento y se negó a trabajar desde el punto de vista familiar, no se pueden aportar datos en este ámbito, aunque cualitativamente destaca que mejora la relación con la madre de su hijo y retoma las responsabilidades parentales. Asimismo, se observaron mejorías en sus recursos psicológicos como la inteligencia emocional y las habilidades sociales (tabla 2).

La alianza terapéutica (Inventario de Alianza Terapéutica y Teoría de Cambio, WATOCI) (Corbella \& Botella, 2004) es un buen indicador de la efectividad del proceso terapéutico, obteniendo buenos resultados en: acuerdo de las tareas de la terapia (28), vínculo emocional entre terapeuta y paciente (22), acuerdo en los objetivos (28) y teoría del cambio (35).

Por otro lado, también se observaron mejorías en los instrumentos administrados al terapeuta (tabla 2): las puntuaciones del CGI-SI y del EEAG fueron 5 (marcadamente enfermo) y 41 (síntomas graves) en el pretratamiento, 2 (moderadamente mejor) en el CGI-GI y 61 (algunos síntomas leves o alguna dificultad psicosocial) en el EEAG, ya que, a lo largo del seguimiento, $\mathrm{M}$ refiere la persistencia de algunos síntomas residuales de rituales obsesivo-compulsivos.

La situación vital después del tratamiento de $\mathrm{M}$ cambió radicalmente, mientras que al inicio $\mathrm{M}$ solo jugaba a videojuegos y apenas prestaba atención a buscar trabajo o a atender a su hijo, al final del tratamiento se responsabilizó de sus funciones parentales y de colaborar en el hogar de los padres. Asimismo, se activó desde el punto de vista laboral y encontró trabajo. Por otro lado, retomó las aficiones musicales que dejó a causa de su adicción.

Cualitativamente, el paciente verbalizó algunas indicaciones sobre los resultados terapéuticos: «quien me habría dicho que al final podría controlar el tema de los videojuegos y ya no jugaría», «ahora estoy infinitamente mejor», «tengo muchas menos manías, lo ideal para mí sería que desaparecieran por completo, si no tuviera estas manías ya sería perfecto, es lo único que me falta conseguir», «me noto mejor, con más ganas de hacer cosas, antes nada me motivaba, ahora tengo trabajo y hago cosas importantes para mí», «todo es gracias al esfuerzo personal que he hecho y al venir aquí».

\section{Discusión}

En el presente estudio se ha descrito un caso piloto de tratamiento del Internet Gaming Disorder mediante el programa PIPATIC. A pesar de la rigidez que comporta la realización de un estudio de caso con un tratamiento prediseñado experimental, fue posible realizar pequeñas adaptaciones que no modificaron la estructura del programa y que se adaptaron a las necesidades de $\mathrm{M}$.

Los resultados indican que los objetivos terapéuticos del programa de tratamiento PIPATIC se cumplieron, reduciendo los síntomas clínicos, los hábitos de uso desadaptativos y los problemas de conducta. Por otro lado, se observó una mejoría de los recursos psicológicos, autoestima, inteligencia emocional, habilidades sociales y funcionamiento global. Sin embargo, todavía presenta sintomatología residual de TOC en remisión. Por otro lado, se han producido una serie de mejoras que no quedan reflejadas en las pruebas aplicadas: a) aumento del insight, flexibilidad cognitiva, capacidades de afrontamiento adecuadas, capacidad de adaptarse a 
las situaciones y reducción de cogniciones inadecuadas que mantenían el problema; b) mejora en las áreas laboral, familiar, relaciones interpersonales y ocio; c) concienciación sobre la posibilidad de recaer y sobre cómo afrontar posibles recaídas; y d) se ha generado un cambio importante en múltiples funcionamientos desadaptativos, por ejemplo, $\mathrm{M}$ es capaz de adaptarse y gestionar la frustración. Este caso sirve para ilustrar que el Internet Gaming Disorder, habitualmente, se acompaña de otras problemáticas comórbidas que deben ser tratadas para generar un avance en el proceso psicoterapéutico, ya que algunos síntomas promueven el mantenimiento de otros (por ejemplo, la falta de autocontrol producía mucha ansiedad en $\mathrm{M}$ ).

El presente estudio adolece de las limitaciones propias de un diseño de caso único. A pesar de ello, los resultados obtenidos apuntan a una elevada efectividad de las terapias cognitivo-conductuales (Beranuy et al., 2012; Griffiths \& Meredith, 2009; Hall \& Parsons, 2001; King, Delfabbro, \& Griffiths, 2010; King et al., 2011; Marco \& Chóliz, 2014; Young, 2013), y del PIPATIC en particular, para el uso problemático de videojuegos. Estas mejorías se deben verificar en el seguimiento a los tres meses. Asimismo, y debido a la necesidad de seguir investigando en este campo, se pretende analizar casos de diferentes edades, sexo y contextos, para confirmar y extender los resultados de efectividad terapéutica a distintos perfiles clínicos. Gracias a los resultados prometedores de este estudio piloto, se ha iniciado una investigación para comparar un grupo de pacientes tratados con el programa PIPATIC con un grupo control de terapia cognitivo-conductual estándar.

\section{Agradecimientos}

A M por haber decidido formar parte de este estudio. Este trabajo ha sido posible gracias a una ayudas a la Investigación Ignacio Hernando de Larramendi (Fundación Mapfre).

\section{Referencias}

ADESE. (2009). Usos y hábitos de los videojugadores españoles. Recuperado de http://www.adese.es/pdf/PPThabitos122009.pdf

Alario, S. (2006). Tratamiento de un caso crónico de adicción a internet. Cuadernos de Medicina Psicosomática y Psiquiatría de Enlace, 70(80), 71-88.

Allison, S., Wahlde, L., Shockley, T., \& Gabbard, G. (2006). The Development of the self in the era of the Internet and role-playing fantasy games. American Journal of Psychiatry, 163(3), 381-385.

American Psychiatric Association. (1987). Escala de Evaluación de la Actividad Global-EEAG. In DSM-IIIR: Diagnostic amb Statistical Manuel of Mental Disorders. Washington, DC: American Pysichiatric Association.

American Psychiatric Association. (2013). DSM-5: Diagnostic and Statistical Manual of Mental Disorders. Washington, DC: American Psychiatric Association.
Baumrind, D. (1968). Authoritarian vs. authoritative parental control. Adolescence, 3(11), 255-272.

Beck, A. T. (1979). Cognitive therapy and the emotional disorders. Boston: Penguin.

Beranuy, M., Carbonell, X., \& Griffiths, M. (2012). A qualitative analysis of online gaming addicts in treatment. International Journal of Mental Health and Addiction, 11(2), 149-161. http://doi.org/10.1007/ s11469-012-9405-2

Beranuy, M., Chamarro, A., Graner, C., \& Carbonell, X. (2009). Validación de dos escalas breves para evaluar la adicción a Internet y el abuso de móvil. Psicothema, 21(3), 480-485.

Carbonell, X., Fúster, H., Chamarro, A., \& Oberst, U. (2012). Adicción a internet y móvil: una revisión de estudios empíricos españoles. Papeles Del Psicólogo, 33(2), 82-89.

Carbonell, X., Guardiola, E., Beranuy, M., \& Bellés, A. (2009). A bibliometric analysis of the scientific literature on Internet, video games, and cell phone addiction. Journal of The Medical Library Association, 97(2), 102-107. http://doi.org/10.3163/15365050.97.2.006

Chappell, D., Eatough, V., Davies, M. N. O., \& Griffiths, M. (2006). EverQuest-It's Just a Computer Game Right? An interpretative phenomenological analysis of online gaming addiction. International Journal of Mental Health and Addiction, 4(3), 205-216. http:// doi.org/10.1007/s11469-006-9028-6

Corbella, S., \& Botella, L. (2004). Psychometric properties of the Spanish version of the Working Alliance Theory of Change Inventory (WATOCI). Psicothema, 16(4), 702-705.

Cruzado, L., Matos, L., \& Kendall, R. (2006). Adicción a internet: Perfil clínico y epidemiológico de pacientes hospitalizados en un instituto nacional de salud mental. Revista Médica Herediana, 17(4), 196-205.

Derogatis, L. R. (2002). SCL-90-R: cuestionario de 90 sintomas: manual. Madrid: TEA.

Ellis, A. (1990a). Is rational-emotive therapy (RET) «rationalist» or «constructivist?» In A. Ellis \& W. Dryden (Eds.), The essential Albert Ellis (pp. 114-141). New York: Springer.

Ellis, A. (1990b). Special features of rational-emotive therapy. In W. Dryden \& R. DiGiuseppe (Eds.), A primer on rational-emotive therapy (pp. 79-93). Champaign, IL, US: Research Press.

Ferguson, C. J., Coulson, M., \& Barnett, J. (2011). A meta-analysis of pathological gaming prevalence and comorbidity with mental health, academic and social problems. Journal of Psychiatric Research, 45(12), 1573-1578. http://doi.org/10.1016/j.jpsychires.2011.09.005

Fernández-Berrocal, P. Alcaide, R., Domínguez, E., Fernández-McNally, C. Ramos, N. S., \& Ravira, M. (1998). Adaptación al castellano de la escala rasgo de metaconocimiento sobre estados emocionales de Salovey et al.: datos preliminares. Libro de Actas Del $V$ Congreso de Evaluación Psicológica, 1, 83-84.

Fundación Orange. (2014). eEspaña: Informe Anual sobre 
el desarrollo de la sociedad de la información en España. Fundación Orange. Madrid. Recuperado de http:// www.proyectosfundacionorange.es/docs/eE2014/ Informe_eE2014.pdf

Fuster, H., Oberst, U., Griffiths, M., Carbonell, X., Chamarro, A., \& Talarn, A. (2012). Psychological motivation in online role-playing games : A study of Spanish World of Warcraft players. Anales de Psicología, 28(1), 274-280.

Gentile, D. a, Choo, H., Liau, A., Sim, T., Li, D., Fung, D., \& Khoo, A. (2011). Pathological video game use among youths: a two-year longitudinal study. Pediatrics, 127(2), e319-29. http://doi.org/10.1542/ peds.2010-1353

Gismero, E. (2000). EHS Escala de habilidades sociales. Madrid: TEA.

González, V., Merino, L., \& Cano, M. (2009). Las eadicciones. Dependencias en la era digital: Ciberjuego, cibersexo, comunidades y redes sociales. (Vol. 2009). Barcelona: Nexusmédic.

Greenfield, D. N. (1999). Virtual addiction: Help for netheads, cyberfreaks, and those who love them. Oakland, CA: New Harbin.

Griffiths, M. (2000). Does Internet and computer «addiction» exist? Some case study evidence. CyberPsychology \& Behavior, 3(2), 211-218. http://doi. org/10.1089/109493100316067

Griffiths, M. D., \& Meredith, A. (2009). Videogame addiction and its treatment. Journal of Contemporary Psychotherapy, 39(4), 247-253. http://doi.org/10.1007/ s10879-009-9118-4

Hall, A. S., \& Parsons, J. (2001). Internet addiction: College student case study using best practices in cognitive behavior therapy. Journal of Mental Health Counseling, 23(4), 312-327.

Han, D. H., Lee, Y. S., Shi, X., \& Renshaw, P. F. (2014). Proton magnetic resonance spectroscopy (MRS) in on-line game addiction. Journal of Psychiatric Research, $58,63-68$.

Kadera, S. W., Lambert, M. J., \& Andrews, A. A. (1996). How much therapy is really enough?: A session-bysession analysis of the psychotherapy dose-effect relationship. The Journal of Psychotherapy Practice and Research, 5(2), 132-151.

Kelly, R. V. (2004). Massively multiplayer online roleplaying games: The people, the addiction and the playing experience. Jefferson, North Carolina: McFarland.

King, D., Delfabbro, P., \& Griffiths, M. (2010). Cognitive behavioral therapy for problematic video game players: Conceptual considerations and practice issues. Journal of Cyber Therapy and Rehabilitation, 3(3), 261-273.

King, D., Delfabbro, P., Griffiths, M., \& Gradisar, M. (2011). Assessing clinical trials of Internet addiction treatment: A systematic review and CONSORT evaluation. Clinical Psychology Review, 31(7), 1110-1116. http://doi.org/doi:10.1016/j.cpr.2011.06.009

King, D. L., \& Delfabbro, P. H. (2014). The cognitive psychology of Internet gaming disorder. Clinical Psychology Review, 34(4), 298-308. http://doi. org/10.1016/j.cpr.2014.03.006
Kleinke, C. L. (1995). Principios comunes en psicoterapia. Bilbao: Desclée de Brouwer.

Ko, C.-H., Yen, J.-Y., Chen, S.-H., Wang, P.-W., Chen, C.-S., \& Yen, C.-F. (2014). Evaluation of the diagnostic criteria of Internet gaming disorder in the DSM-5 among young adults in Taiwan. Journal of Psychiatric Research, 53, 103-10. http://doi.org/10.1016/j.jpsychires.2014.02.008

Lambert, M. J., \& Bergin, A. E. (1994). The effectiveness of psychotherapy. Oxford: John Wiley.

Lega, L. I., Caballo, V. E., \& Ellis, A. (2009). Teoría y práctica de la terapia racional emotivo-conductual. Madrid: Editorial Siglo XXI.

Marco, C., \& Chóliz, M. (2014). Tratamiento cognitivoconductual de la adicción a videojuegos de rol online. Anales de Psicología, 30(1), 46-55.

Matalí, J. L., \& Alda, J. A. (2008). Adolescentes y nuevas tecnologías: ¿innovación o adicción? Barcelona: Edebé.

Millon, T., Millon, C., Davis, R. D., \& Grossman, S. (2009). MCMI-III: Millon clinical multiaxial inventoryIII. Pearson.

Moos, R. (1974). The Social Climate Scales: An Overview. California: Palo Alto: Consulting Psychological Press.

ONTSI. (2013). Perfil sociodemográfico de los internautas. Recuperado de http://www.ontsi.red.es/ontsi/sites/ default/files/perfil_sociodemografico_de_los_internautas_2013_0.pdf

Prochaska, J., Norcross, J., \& DiClemente, C. (1994). Changing for good. The revolutionary program that explains the six stages of change and teaches you how to free yourself from bad habits. New York: William Mo.

Salovey, P., Mayer, J., Goldman, S. L., Turvey, C., \& Palfai, T. P. (1995). Emotional attention, clarity and repair. Exploring emotional intelligence using the Trait Meta-Mood Scale. In J. W. Pennebaker (Ed.), Emotion, Disclosure, and Health. (pp. 125-154). Washington: American Psychiatric Association.

Shapira, N. A., Goldsmith, T. D., Keck Jr, P. E., Khosla, U. M., \& McElroy, S. L. (2000). Psychiatric features of individuals with problematic internet use. Journal of Affective Disorders, 57, 267-272.

Torres, A., Carbonell, X., \& Oberst, U. (n.d.). Manual de tratamiento de las adicciones tecnológicas: Programa de intervención psicológica en el uso problemático de las tecnologías de la información y la comunicación en adolescentes y adultos (PIPATIC). Barcelona: Universitat Ramon Lull.

Yee, N. (2006). Motivations for play in online games. Cyberpsychology \& Behavior, 9(6), 772-775. http://doi. org/10.1089/cpb.2006.9.772

Yen, J.-Y., Ko, C.-H., Yen, C.-F., Chen, S.-H., Chung, W.-L., \& Chen, C.-C. (2008). Psychiatric symptoms in adolescents with Internet addiction: Comparison with substance use. Psychiatry and Clinical Neurosciences, 62(1), 9-16. http://doi.org/10.1111/j.1440-1819. 2007.01770.x

Young, K. S. (2007). Cognitive behavior therapy with Internet addicts: treatment outcomes and implications. Cyberpsychology \& Behavior, 10(5), 671-679. http://doi.org/10.1089/cpb.2007.9971 
Young, K. S. (2011). CBT-IA: The first treatment model for internet addiction. Journal of Cognitive Psychotherapy, 25(4), $304-312$.

Young, K. S. (2013). Treatment outcomes using CBT-IA with internet-addicted patients. Journal of Behavioral Addictions, 2(4), 209-215. http://doi.org/10.1556/ JBA.2.2013.4.3

\section{Addicció als videojocs en línia: tractament mitjançant el programa PIPATIC}

Resum. El desenvolupament de les Tecnologies de la Informació i la Comunicació (TIC) ha propiciat investigacions sobre l'addicció a Internet, als videojocs $i$ telèfons mòbils $i$ el seu tractament. L'objectiu del present estudi és presentar l'aplicació d'un programa de tractament psicoterapèutic per l'addicció a les TIC (PIPATIC) i l'avaluació de la seva efectivitat en un estudi de cas únic d'un pacient amb trastorn dual: Internet Gaming Disorder (IGD) i trastorn obsessiu-compulsiu (TOC). Els resultats són encoratjadors i mostren canvis positius: reducció significativa del temps dedicat als jocs, augment de l'autocontrol i reducció de la simptomatologia general $i$ dels simptomes relacionats amb el TOC.

Paraules clau: trastorn de joc per Internet; trastorn obsessiucompulsiu; addicció a videojocs; patologia dual; tractament; estudi de cas 
